\title{
Detection of efficient carrier capture in ultrathin InAs/GaAs layers using a degenerate pump-probe technique
}

\author{
Bo Liu ${ }^{1,3}, \mathbf{Q ~ L i}{ }^{1,4}$, Zhongying $\mathrm{Xu}^{1}$ and $\mathrm{W} \mathrm{K} \mathrm{Ge}^{2}$ \\ ${ }^{1}$ National Laboratory of Supperlattices and Microstructures, Institute of Semiconductors, \\ Academia Sinica, Beijing 100083, China \\ 2 Department of Physics, Hong Kong University of Science and Technology, Kowloon, \\ Hong Kong \\ E-mail: liubo@red.semi.ac.cn
}

Received 25 February 2001, in final form 1 March 2001

\begin{abstract}
By analysing the carrier dynamics based on the rate equations and the change of the refractive index due to the efficient carrier capture, we have calculated the carrier capture process in the InAs/GaAs system detected by a simple degenerate pump-probe technique. The calculated results are found to be in good agreement with the experimental findings. Our results indicate that this simple technique, with the clear advantage of being easy to carry out, can be very useful in studying the carrier dynamics for some specific structures such as InAs ultrathin layers embedded in a GaAs matrix described here.
\end{abstract}

Carrier capture and relaxation processes in InAs/GaAs quantum structures have attracted much attention due to wide physical interest [1-3] and potential device applications [4-7]. There have been a number of experiments [8-10] concerning the carrier dynamics in self-organized InAs/GaAs quantum dots (QDs) over the past few years. Various experimental methods were employed in the experiments, including the complex multiple-wavelength pump-probe technique [8], delicate up-conversion [9] and the high resolution streak camera [10]. The carrier relaxation in InAs/GaAs QDs was found to occur as fast as in higher dimensional systems, on a time scale of several picoseconds. In our previous paper [11], we demonstrated that the carrier capture process in InAs/GaAs quantum dots could be detected by a simple degenerate pump-probe technique even if the probe energy is much higher than the exciton energy levels of the InAs/GaAs system. The measured capture time was found to depend on the InAs layer thickness and the excitation density as well.

In this paper we further simulate the carrier capture process by analysing the carrier dynamics based upon the rate equations and the change of the refractive index due to carrier injection according to the Kramers-Kronig relations. The calculated results were found to be

3 Corresponding author.

4 Present address: Department of Physics, Hong Kong University. 
fairly consistent with the experimental data, showing the validity of our simple experimental method.

The samples in the experiments were grown on (001) GaAs substrates by molecular-beam epitaxy. A $0.5 \mu \mathrm{m} \mathrm{GaAs}$ buffer layer was deposited first at $580^{\circ} \mathrm{C}$, followed by a single InAs layer with different thickness grown at $450{ }^{\circ} \mathrm{C}$. Then a $20 \mathrm{~nm} \mathrm{GaAs}$ cap was grown on the top at the same substrate temperature. A degenerate pump-probe technique operating in a reflectance geometry was performed with a mode-locked Ti:sapphire laser. The intensity ratio between pump and probe beams is approximately 10:1, and the two beams are orthogonally polarized in order to minimize the contribution from the coherent artifact. The initial photoinjected carrier density is estimated to be around $1 \times 10^{17} \mathrm{~cm}^{3}$. The probe (pump) energy in our experiments was chosen to be $1.55 \mathrm{eV}$, which is much higher than the exciton energy levels of the InAs/GaAs samples. The details of our experiments can be found elsewhere [11].

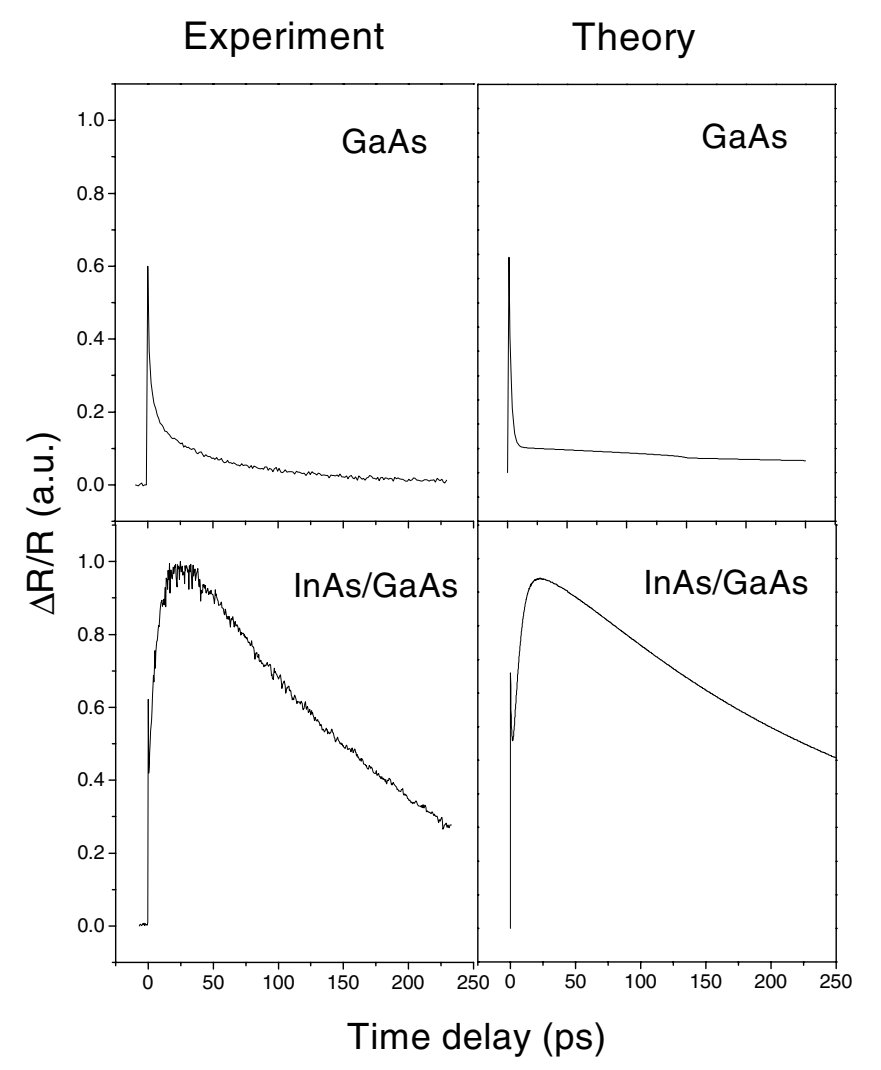

Figure 1. Transient differential reflectance, $\Delta R / R$ against time delay, for two different samples at $77 \mathrm{~K}$. Two samples were excited and probed at $1.550 \mathrm{eV}$. Note that the experimental results are well comparable to the model calculation.

Figure 1 shows two typical transient differential reflectances for two different samples: one is $1 \mathrm{ML}$ InAs/GaAs and another is a bulk GaAs reference sample. The samples were excited at $1.550 \mathrm{eV}$, i.e. $40 \mathrm{meV}$ above the band-edge of $\mathrm{GaAs}$ at $77 \mathrm{~K}$. As expected, the transient reflectance for the GaAs reference sample increases and then drops very fast, corresponding to the build-up of photogenerated carriers and the subsequent fast thermalization and recombination [12]. However, for the InAs/GaAs sample, an additional rise in the 
reflectance was observed following the similar initial peak. The second peak has been attributed to the efficient carrier capture from the GaAs barriers into the InAs layer [11]. By fitting the rising process with an exponential function, the time constant of 6.3 ps was obtained.

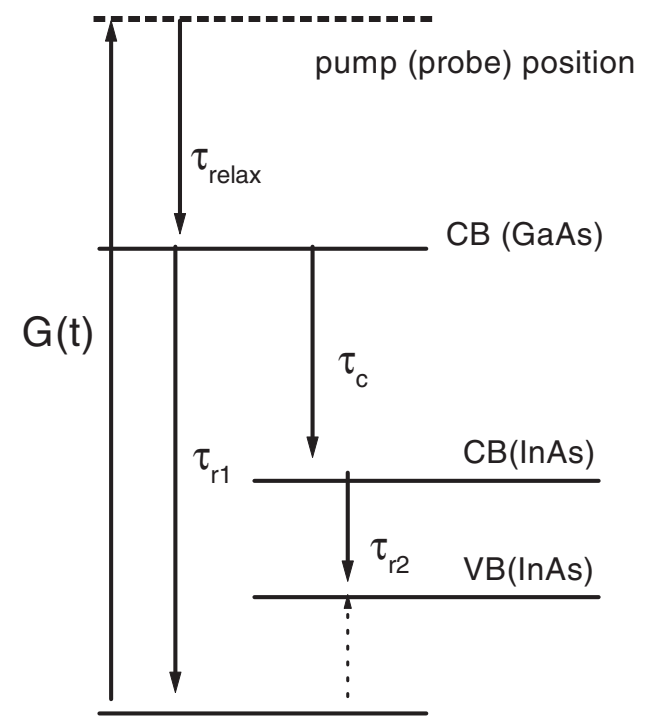

Figure 2. Schematic presentation of the carrier dynamic processes involved in the rate equations for the InAs/GaAs heterostructure.

In order to well understand the experimental results, we have developed rate equations to model the carrier dynamics in the InAs/GaAs structure. The processes involved in the model are indicated in figure 2, and can be described by the following set of differential equations:

$$
\begin{aligned}
& \frac{\mathrm{d} n_{p}(t)}{\mathrm{d} t}=G(t)-\frac{n_{p}(t)}{\tau_{\text {relax }}} \\
& \frac{\mathrm{d} n_{1}(t)}{\mathrm{d} t}=\frac{n_{p}(t)}{\tau_{\text {relax }}}-\frac{n_{1}(t)}{\tau_{r 1}}-\frac{n_{1}(t)}{\tau_{C}} \\
& \frac{\mathrm{d} n_{\text {InAs }}(t)}{\mathrm{d} t}=\frac{n_{1}(t)}{\tau_{C}}-\frac{n_{\text {InAs }}(t)}{\tau_{r 2}}
\end{aligned}
$$

where $n_{p}, n_{1}$ are the carrier (electron) populations at the pump energy position and at the edge of the conduction band of GaAs, respectively. $n_{\text {InAs }}$ is the electron population in the InAs layer. $G(t)$ is the generation rate for a Gaussian laser pulse with width about $80 \mathrm{fs} . \tau_{r 1}$ and $\tau_{r 2}$ are the electron decay times in the GaAs barrier and InAs layer, and $\tau_{C}$ the electron capture time from GaAs into InAs layer. We neglect the hole population process in the system (denoted as a dotted arrow in figure 2) due to the fast hole capture process from GaAs into InAs layer, as it will not appear in the time dependence of the optical parameters to be studied. It is worth noting that the photoexcited carriers are created primarily in the GaAs matrix surrounding the thin InAs layer. The InAs layer may have ground as well as excited states, and the dynamics of carrier in InAs/GaAs system following the photoexcitation is expected to be complicated $[9,13,14]$. Here, however, we are mainly concerned with the change of the total carrier density and the sequential changes in the absorption and refractive index far above the bandgap of InAs layer; the role of excited states versus ground states is not relevant in the discussion. 


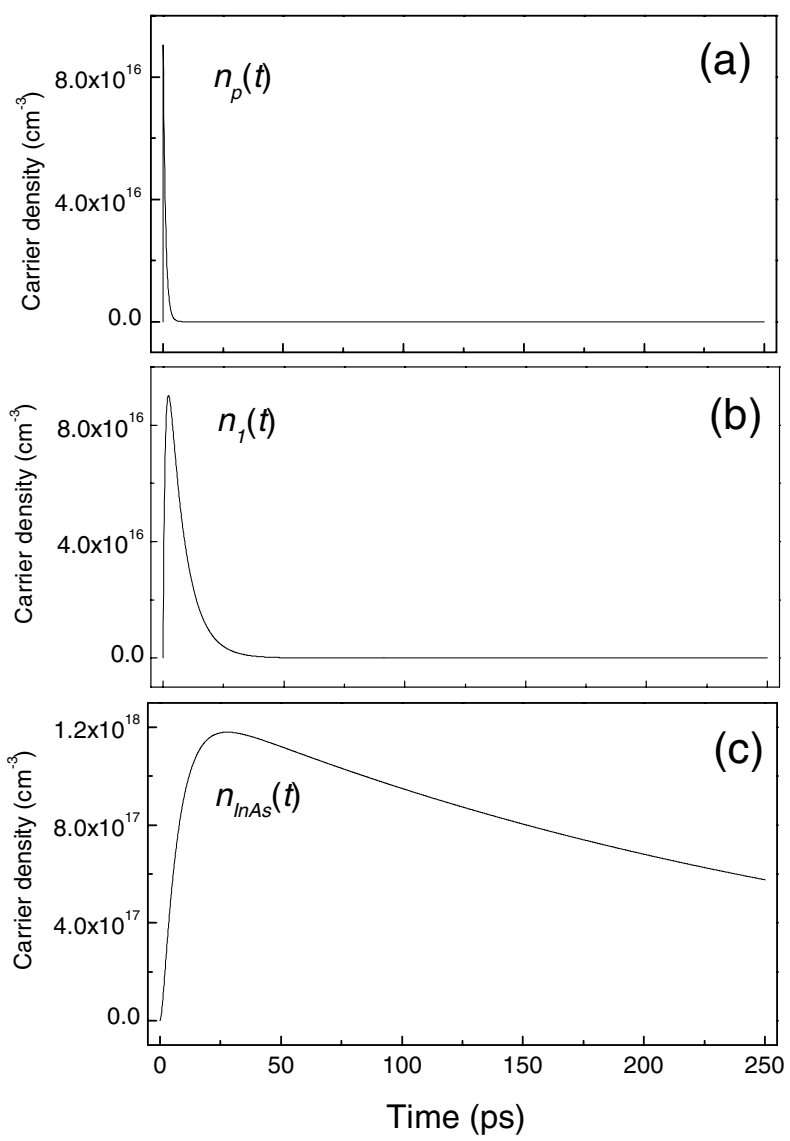

Figure 3. Temporal evolution of the carrier density at (a) the pump (probe) energy, $n_{p}(t)$, (b) the bottom of the conduction band of GaAs, $n_{1}(t)$, and (c) the conduction band of InAs, $n_{\operatorname{InAs}}(t)$, respectively.

Figure 3 shows the calculated temporal evolution of the carrier density at (a) the pump (probe) energy, (b) the bottom of the conduction band of GaAs and (c) the bandgap of InAs, respectively. In the calculation the following parameters were used: the initial excitation carrier density is $1.0 \times 10^{17} \mathrm{~cm}^{-3}$, the relaxation time of photogenerated carriers in GaAs $2 \mathrm{ps,}$ the decay time $1 \mathrm{~ns}$ in GaAs and the decay time $250 \mathrm{ps}$ for the $1 \mathrm{ML}$ InAs/GaAs (measured by time-resolved PL). The carrier capture time of the InAs layer from the GaAs barrier is taken as an adjustable parameter, being $\tau_{C}=7 \mathrm{ps}$ in our calculation. From figure 3 one can see that with almost no delay, the absorption of the pump pulse in GaAs places carriers high in the conduction band, where they then rapidly relax down in a time scale typically of 1-2 ps. This leads to a rapid increase of the carrier density in the bottom of the conduction band. The carrier density then decays away due to the efficient capture of the InAs layer, as well as the recombination in GaAs. The efficient capturing process in the InAs layer is evidenced by the increase of the carrier density in InAs layer (figure 3(c)). From the calculation, it is also found that the carrier density in the thin InAs layer can be enhanced due to the efficient carrier capturing. The maximum carrier density reaches $\sim 1.3 \times 10^{18} \mathrm{~cm}^{-3}$ as shown in figure 3(c), much higher than the initial injection carrier density of $1 \times 10^{17} \mathrm{~cm}^{-3}$. This is very important 
for understanding the significant change of the refractive index far above the bandgap of the InAs layer. It is also noted that the above carry density is several orders of magnitude larger than the quantum dot density in the case of InAs QDs. However, considering that the whole InAs layer is usually composed of a 2D wetting layer and conelike InAs islands, such a high carrier density can indeed be achieved.

It is known that in the pump-probe measurement, the photo-generated carriers induce nonlinear absorption variations through the mechanisms of bandfilling, bandgap renormalization and free carrier absorption [15]. Any change in the absorption coefficient $\Delta \alpha$ is accompanied by a change in the refractive index $\Delta \eta$, which then depends on the probe energy as well as the photo-generated carrier density. For the photo-generated carrier densities typically used for reflectivity measurements $\left(\leqq 10^{18} \mathrm{~cm}^{-3}\right)$, the electron bandfilling dominates the non-linear refractive index changes at the photon energies higher than the bandgap. The change of the refractive index at the probe energy $E_{p}$ can be expressed using the KramersKronig relations [15]:

$$
\Delta \eta(E-p, t) \propto P \int_{0}^{\infty} \frac{\Delta \alpha(E, t)}{E^{2}-E_{p}^{2}} \mathrm{~d} E
$$

where $E_{p}=\hbar \omega$ is the probe photon energy.

In the case for the bulk GaAs, only the absorption process in GaAs contributes to $\Delta \eta$. However, in the case for the InAs/GaAs system, the efficient carrier trapping by the thin InAs layer will produce a large carrier density in the InAs layer, as presented in figure 3(c); therefore, great changes in the absorption coefficient and refractive index are expected.

To further establish the absorption formula in the InAs layer, we treat the thin InAs layer embedded in GaAs as a quantum well [16]. Then, the absorption in the InAs layer can be expressed as $[15,17]$ :

$$
\alpha(\omega)=\frac{C}{\omega} \Theta\left(E_{p}-E_{g}\right)=\frac{C_{h h}^{2 d}+C_{l h}^{2 d}}{\omega} \Theta\left(E_{p}-E_{g}\right)
$$

and

$$
\Delta \alpha(\omega)=\frac{C}{\omega} \Theta\left(E_{p}-E_{g}\right) A(\omega)
$$

where $E_{g}$ is the ground energy level of the InAs layer $(1.43 \mathrm{eV}$ for a $1 \mathrm{ML}$ InAs/GaAs sample according to PL measurement), and $\Theta$ is a step function. $A(\omega)=\left[f_{v}(\omega)-f_{c}(\omega)-1\right]$ is a bandfilling factor. $C$ is a constant involving material parameters and matrix elements between the Bloch functions at the band edges [15].

Using the above equations the changes of refractive index $(\Delta \eta)$ under different injection carrier densities in the InAs layer have been calculated. Figure 4 displays the calculated changes of the refractive index under different injection carrier densities in the InAs layer. It can be seen that the calculated refractive index decreases for the energies near and below $E_{g}$ and $\Delta \eta$ becomes positive for the energies well above $E_{g}$. This indicates that the high carrier density due to the efficient carrier trapping in InAs layer has a significant effect on the change of the refractive index even at the energy position well above the energy level of InAs. In other words, in the degenerate pump-probe measurement, although the probe energy is much higher than the energy level for the detection (InAs bandgap in our case), it can still sense the carrier dynamics at that detection energy. For example, for a carrier density of $1.3 \times 10^{18} \mathrm{~cm}^{-3}$ in the InAs layer, the change of the refractive index at energy position of $1.55 \mathrm{eV}$ (the probe energy in figure 1) due to the efficient carrier trapping in the InAs layer is $\sim 0.06$, which further results in the change of the reflectance of around $1 \%$. This valve is comparable to that observed in the experiment. 


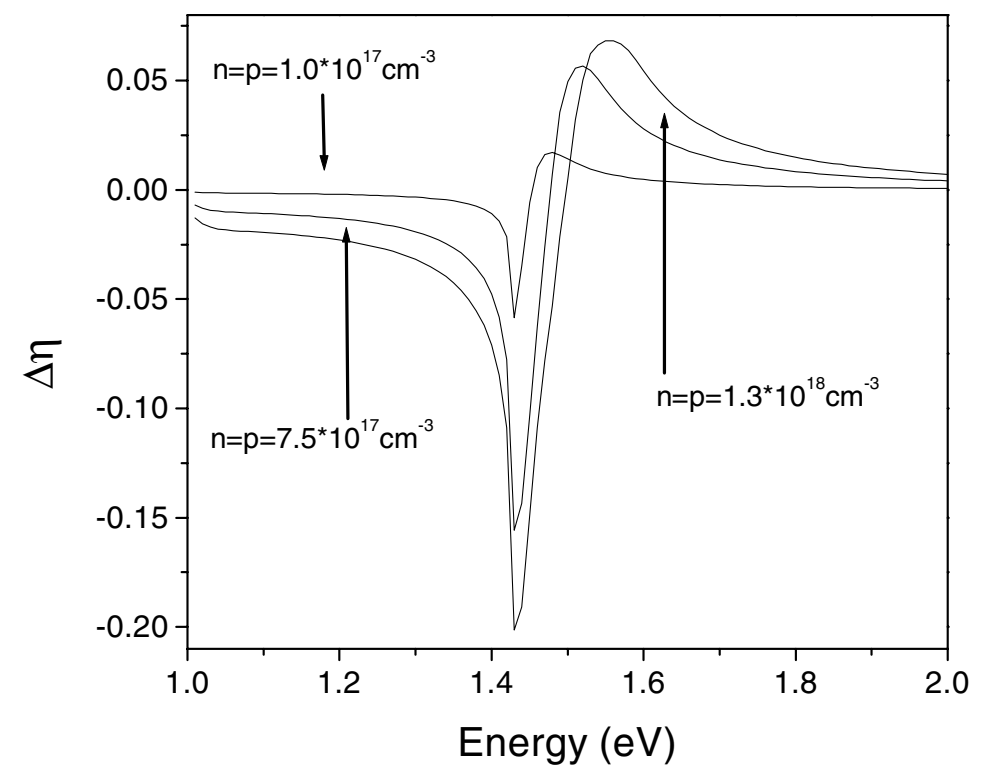

Figure 4. Calculated changes of the refractive index against energy under different carrier densities in the InAs layer, showing that the high carrier density in InAs layer has a significant effect on the change of the refractive index even at an energy position well above the bandgap.

In order to further compare with the experimental results, we have calculated the derivative of the reflectance, $\Delta R / R$, using equation (7). The detailed derivation of the equation is given in the appendix

$$
\frac{\Delta R}{R} \approx \frac{4}{\left(\eta_{G a A s}^{2}-1\right)} \Delta \eta_{G a A s}+\frac{32 n_{G a A s}^{2} \cos (2 k d)}{\left(\eta_{G a A s}^{2}-1\right)\left(\eta_{G a A s}+\eta_{I n A s}\right)^{2}} \Delta \eta_{I n A s} .
$$

Equation (7) clearly indicates that there are two contributions to the change of the reflectance. The first term, that gives rise to the sharp peak near zero delay in the transient reflectance, is the contribution from the GaAs matrix. The second term corresponds to the contribution from the InAs layer, which induces the additional peak in the transient reflectance. Using the same parameters as in the previous calculation, we have calculated the derivative of the reflectance for the two samples. The results are shown in the left side of figure 1. It is found that the model calculation and the experiments are in surprisingly good agreement, considering the approximations used in the deriving the model. We therefore conclude that the dramatic change of the carrier density in InAs layer can indeed produce a significant change of the reflectivity well above the InAs energy level.

In conclusion, we have investigated the carrier capture process in the InAs/GaAs thin layers by a degenerate pump-probe technique. By analysing the carrier dynamics based on the rate equations and the change of the refractive index due to the efficient carrier capturing using the Kramers-Kronig relation, the carrier capture process in InAs/GaAs system is well simulated. Our results indicate that this simple technique, with the clear advantage of being easy to carry out, can be very useful in studying the carrier dynamics for some specific structures, such as InAs ultrathin layers embedded in a GaAs matrix described here, although it is usually thought the interpretation of such an experiment is quite complex. 


\section{Acknowledgments}

The project is supported by grant numbers 69736010 and 19574047 from the National Natural Science Foundation of China and grant HKUST 6135/97P from RGC, Hong Kong. We wish to thank Dr Yuan at Clarendon, Oxford for valuable discussions.

\section{Appendix}

For simplicity, we first treat our InAs/GaAs heterostructure as a model structure, in which an InAs thin layer is sandwiched between a GaAs layer with thickness $d$ and refractive index $n$ and a semi-infinite GaAs substrate of the same refractive index. The InAs layer is characterized by the reflection coefficient $r_{\text {InAs }}$. Then the reflectivity $R$ can be written as [18]

$$
R=\left|\frac{r_{0}+r_{I n A s} \mathrm{e}^{\mathrm{i} 2 k d}}{1+r_{0} r_{I n A s} \mathrm{e}^{\mathrm{i} 2 k d}}\right|^{2}
$$

where the surface reflection coefficient $r_{0}=\left(1-\eta_{G a A s}\right) /\left(1+\eta_{G a A s}\right)$, the wavevector $k=2 \pi \eta_{G a A s} / \lambda$, the InAs layer reflection coefficient $r_{I n A s} \approx r^{\prime}-T T^{\prime} r^{\prime} \exp [\mathrm{i}(\pi+\varphi)]$ and the coefficients of the two surfaces of the InAs layer $T=\left(2 \eta_{G a A s}\right) /\left(\eta_{G a A s}+\eta_{I n A s}\right)$ and $T^{\prime}=\left(2 \eta_{I n A s}\right) /\left(\eta_{G a A s}+\eta_{I n A s}\right)$, respectively. The surface reflection coefficient of the InAs layer $r^{\prime}=\left(\eta_{\text {GaAs }}-\eta_{\text {InAs }}\right) /\left(\eta_{G a A s}+\eta_{I n A s}\right)$, the relative phase of the rays on a plane outside the InAs layer perpendicular to the ray changes by $\varphi=4 \pi d^{\prime} \eta_{\operatorname{InAs}} / \lambda, d^{\prime}$ is the thickness of the InAs layer.

Taking the derivative of equation (A.1), the differential reflectance is written as follows:

$$
\begin{aligned}
\frac{\Delta R}{R}=\frac{\left[2\left(1-r_{0}^{2}\right)\right]\left[\left(1+r_{0}^{2}\right) r_{I n A s}+r_{0}\left(1+r_{I n A s}^{2}\right) \cos (2 k d)\right]}{\left[1+2 r_{0} r_{I n A s} \cos (2 k d)+r_{0}^{2} r_{I n A s}^{2}\right]\left[r_{0}^{2}+2 r_{0} r_{I n A s} \cos (2 k d)+r_{I n A s}^{2}\right]} \Delta r_{I n A s} \\
+\frac{\left[2\left(1-r_{I n A s}^{2}\right)\right]\left[\left(r_{0}\left(1+r_{I n A s}^{2}\right)+\left(1+r_{0}^{2}\right) r_{I n A s} \cos (2 k d)\right)\right]}{\left[1+2 r_{0} r_{I n A s} \cos (2 k d)+r_{0}^{2} r_{I n A s}^{2}\right]\left[r_{0}^{2}+2 r_{0} r_{I n A s} \cos (2 k d)+r_{I n A s}^{2}\right]} \Delta r_{0} .
\end{aligned}
$$

In our experiments, $T T^{\prime} \approx 1, \varphi \approx 0, r_{I n A s} \ll r_{0}$, therefore the reflection coefficient of the InAs layer can be expressed as $r_{I n A s} \approx 2 r^{\prime}$ and then equation (A.2) is simplified as

$$
\frac{\Delta R}{R} \approx \frac{4\left(1-r_{0}^{2}\right) \cos (2 k d)}{r_{0}} \Delta r^{\prime}+\frac{2}{r_{0}} \Delta r_{0}
$$

Referring back to the form of $r_{0}$ and $r^{\prime}$, equation (A.3) could be further written as

$$
\frac{\Delta R}{R} \approx \frac{4}{\left(\eta_{G a A s}^{2}-1\right)} \Delta \eta_{G a A s}+\frac{32 \eta_{G a A s}^{2} \cos (2 k d)}{\left(\eta_{G a A s}^{2}-1\right)\left(\eta_{G a A s}+\eta_{I n A s}\right)^{2}} \Delta \eta_{I n A s}
$$

where $\Delta \eta$ represents the change of the refractive index.

\section{References}

[1] Marzin J Y, Gérald J M, Izräel A, Barrier D and Bastard G 1994 Phys. Rev. Lett. 73716

[2] Leonard D, Krishnamurthy M, Reaves C M, Densaars S P and Petroffr P M 1993 Appl. Phys. Lett. 633203

[3] Xu Z Y, Lu Z D, Yang X P, Yuan Z L, Zheng B Z, Xu J Z, Ge W K, Wang Y, Wang J and Chang L L 1996 Phys. Rev. B 5411528

[4] Ledentsov N N, Krestinikov I L, Maximov M V, Ivanov S V, Sorokin S L, Kop'ev P S, Alfernov Zh I, Bimberg D and Sotomayor Torres C M 1996 Appl. Phys. Lett. 691343

Ledentsov N N, Krestinikov I L, Maximov M V, Ivanov S V, Sorokin S L, Kop'ev P S, Alfernov Zh I, Bimberg D and Sotomayor Torres C M 1997 Appl. Phys. Lett. 702766

[5] Bimberg D, Grundmann M and Ledentsov N N 1998 Quantum Dot Heterostructure (New York: Wiley) 
[6] Goñi A R, Stroh M, Thomsen C, Heinrichsdorff F, Türck V, Krost A and Bimberg D 1998 Appl. Phys. Lett. 72 1433

[7] Ledenstov N N et al 1999 Appl. Phys. Lett. 74161

[8] Sosnowski T S, Norris T B, Jiang H, Singh J, Kamath K and Bhattacharya P 1998 Phys. Rev. B 57 R9423

[9] Yuan Z L, Foo E R A D, Ryan J F, Mowbray D J, Skolnick M S and Hopkinson M 1999 Physica B 27212

[10] Ohensorge B, Albrecht M, Oshinowo J, Forchel A and Arakawa Y 1996 Phys. Rev. B 5411532

[11] Li Q, Xu Z Y and Ge W K 2000 Solid State Commun. 115105

[12] Shah J 1996 Ultrafast Spectroscopy of Semiconductors and Semiconductor Nanostructures (Springer Series in Solid-State Sciences 115) ed M Cardona et al (Berlin: Springer) ch 3

[13] Sandmann J H H, Grosse S, Vonplessen G, Feldmann J, Hayes G, Phillips R, Lipsanen H, Sopanen M and Aphopelto J 1997 Phys. Status Solidi b 204251

[14] Bockelmann U, Roussignol Ph, Filoramo A, Heller W, Abstreiter G, Brunner K, Böhm G and Weimann G 1996 Phys. Rev. Lett. 763622

[15] Bennett B R, Soref R A and del Alamo J A 1990 IEEE J. Quantum Electron. 26113

[16] Alonso M I and Ploog K H 1994 Phys. Rev. B 501628

[17] Haug H and Koch S W 1993 Quantum Theory of the Optical and Electronic Properties of Semiconductors 2nd edn (Singapore: World Scientific)

[18] Malpuech G, Kavokin A, Leymarie J, Disseix P and Vasson A 1999 Phys. Rev. B 6013298 